\title{
An Intelligent Solid Waste Management System using Internet of Things (IoT)
}

\author{
Pramod Mathew Jacob, Rasha Kabeer, Negha Nair, Sony V Saji, Meenakshi, Renju Rachel \\ Varghese
}

\begin{abstract}
Waste Management is one of the hectic and troublesome tasks which require much man power. The traditional waste management system is time consuming and not cost effective. In this scenario, we are designing an intelligent system for solid waste management using Internet of Things. Our proposed system consists of an intelligent smart bin and a mobile application. The smart bin consists of a unique identifier (RFID tag) and an ultra-sonic sensor to determine the waste level of the bin. The mobile application is intended for two user categories: Normal user and Garbage collector. The normal user can locate the nearby dustbins whereas the garbage collector can remotely assess the bin level. When the bin is full, an alert message is sent to the garbage collector and also displays the shortest route to the bin location. It uses genetic algorithm for finding the optimized path from the user to smart bin. The entire connected components and communication is managed by a central coordinator. Our implementation and evaluation results claims that this model can be used as an alternative for the traditional waste management system. This system also ensures cost effectiveness and less time consumption.
\end{abstract}

Index Terms: Internet of things (IoT), Smart waste management, Android application, Smart bin

\section{INTRODUCTION}

Waste management is one of the challenges exist in any country or industry. Various sources of waste include home, industry, organization, restaurants and wherever there is human living. It will exist as a part of everyday activities till life exists. Waste management is a developing issue in both global and local levels. Waste management consists of various processes and activities for managing and disposing wastes effectively. The waste management process includes

phases like collection of garbage, transporting from source to treatment plant and the proper disposal.

The traditional waste management system consists of various bins placed at different locations. People used to

Revised Manuscript Received on July 05, 2019.

Pramod Mathew Jacob, Assistant Professor, Department of Computer Science \& Engineering, Providence College of Engineering, Chengannur, Kerala, India.

Rasha Kabeer, UG Scholar, Department of Computer Science \& Engineering, Providence College of Engineering, Chengannur, Kerala, India. Negha Nair, UG Scholar, Department of Computer Science \& Engineering, Providence College of Engineering, Chengannur, Kerala, India Sony V Saji, UG Scholar, Department of Computer Science \& Engineering, Providence College of Engineering, Chengannur, Kerala, India.

Meenakshi, UG Scholar, Department of Computer Science \& Engineering, Providence College of Engineering, Chengannur, Kerala, India.

Renju Rachel Varghese, Assistant Professor, Department of Computer Science \& Engineering, Providence College of Engineering, Chengannur, Kerala, India. deposit their garbage in the bins. Some garbage collecting person empties the bin by collecting the wastes and transport it to the treatment plant. The treatment plant either disposes or recycles the garbage. The disadvantage of this system is that the person needs to wander here and there to locate a waste bin to deposit the garbage. Also when the waste collector travelled all the distance from the treatment plant to the bin location for collection, there may be a chance that the bin may not be full or even empty. This may not be economical (fuel cost is high) and is a total waste of time. Another challenge is the chance of occurring health problems due to the improper management of waste.

So our proposed model aims to answer the following queries.

\section{Q1: How a user can identify the nearest waste bin?}

Q2: How to manage the transport of waste in an efficient and economical manner?

Q3: How can we remotely monitor the level of waste in a particular bin?

We have studied and evaluated various existing waste management systems. The summary of these studies are provided in the next section. Our proposed system uses an efficient and economical model for solid waste management. The proposed system framework is shown in Figure 1. Our system consists of a Passive Infrared (PIR) motion sensor to detect the presence of a human (user), and automatically opens the lid of the dustbin.

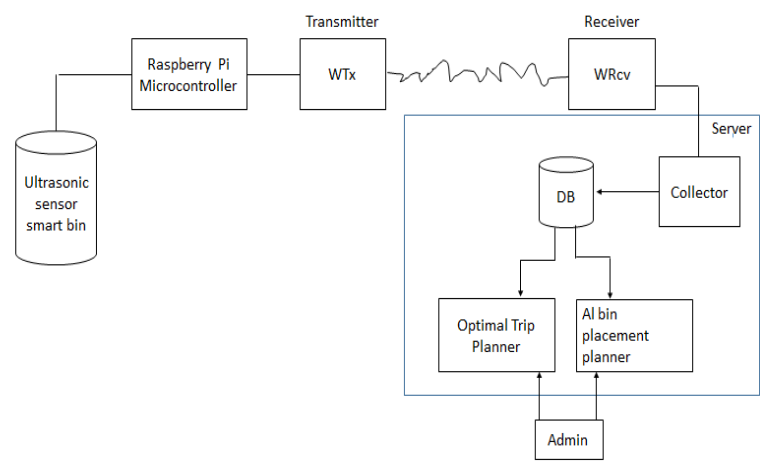

Figure 1: Proposed System Framework

Our mobile application will help the user to identify the locations (GPS coordinates) 
of the nearby dust bins. An ultrasonic sensor is used to measure the bin level. When the waste in the bin reaches a particular threshold, an alert message is sent to the garbage collector. The optimized path from the garbage collector to the dust bin and from dust bin to the treatment plan is generated. Thus our proposed system is an automated system model or architecture for detecting, monitoring and management of solid waste to the server side using GSM in an efficient way.

\section{RELATED WORKS}

We have analyzed about 20 related works in this area and the summary of all is provided below. The analytical and detailed study shows that there exist a lot of gap between the theoretical models and real time models.

Sahil Mirchandani et al [1] proposed an IOT enabled waste bin. Here they use the RFID tags for tracking the wastes and it connected with web based online system and based on the weight of the waste the status of the bin is updated and gives notification when the dustbin is full. And also provide an optimal path for the collection of wastes. Harshita Chugh et al [2] proposed an IOT based smart waste bin which can monitor the waste through sensors. The sensors from different places are connected through internet. The sensors would measure and calculated the amount waste. Also with the help of an android app both the user and authority could got the status of the bins.

Chandradeep Tiwari et al [3] developed a smart solar bin. Here they use two bins one for crush the bio degradable waste and the other for store the bottles and tins. And a wireless technology is used to find the status of the bin, send notifications and route optimization. The modules crushed the waste and compressed them to reduce the volume so thereby that provide space for larger amount of wastes and reduce the number of service for the waste collection. S.Karthikeyan et al [4] proposed a ZigBee network and MQTT (Message Queue Telemetry Transport) protocol for find the status of the bin. The main aim of the proposed system was to find the status of the waste bin and send these data through a wireless ZigBee network. With the help of MQTT a connection link was established between the node and server. Also the travelling salesman algorithm was used to find the optimal distance. And telegramming messaging application was used to find the trucks.

B.S.Malapur et al [5] proposed an automated consist of four modules bin addition, view bin status, collection planning and $\log$. An ultrasonic sensor was used to calculate the status of the bin. Also an optimal path was used for the collection of waste. Ravale et al [6] proposed a solid waste management system which forwards the waste to various biodegradation plants based on waste categories. It alerts the administrator when the bin is full and thereby avoids overflow.

Jayalakshmi et al [7] proposed a novel approach for food waste management through measuring and displaying the amount of food wasted and recycling the wasted food using embedded systems to make fertilizers at planting. The system uses three infrared sensors and also a weight sensor to detect the amount of garbage in the dustbin. Communication takes place through a Wi- Fi module. Ghadage et al [8] review various solid waste management techniques and proposed a system which will take proper care of processing of garbage. The proposed system uses GSM module and ultrasonic sensors to sense the level of garbage in the bin, flame sensor to detect the fire and moisture sensor to separate out wet and dry garbage.

Aleyadeh et al [9] suggests an IoT system for monitoring waste volume as well as routing and scheduling of waste collection trucks based on bin locations. The system uses ultrasonic sensing device to detect the bin level and a humidity sensor to detect the dryness level of the bin content. Abhimanyu Singh et al [10] proposed an IoT garbage management system which uses infrared sensors to detect waste level at a particular period of time. The communication is initiated using Raspberry PI which acts as central coordinator.

Anupama et al [11] suggests a model to measure and sense the water quality level of contaminated water using $\mathrm{pH}$ sensor. The model is good for environmental friendly regions. Mustafa et al [12] suggests a model for municipal waste collectors to remotely sense the garbage content in a particular bin. The garbage level in the bin is measured using ultrasonic sensor and an ARM microcontroller. Every modules are connected to ThingSpeak. Cyril et al [13] proposes an intelligent bin which alerts the authorities only when the bin content is full. It provides the optimized route map to the truck drivers by covering only the route where the bin is full. Neetha et al [14] also proposed a similar system; when the garbage level in bin reaches the threshold value, the bin status is alerted to the authorities by updating it in a cloud database. This can be accessed by the authorities and can take suitable disposal measures in an efficient and economic manner.

Medvedev et al [15] proposed a smart city waste collection using Top-k query based dynamic scheduling algorithm. It analyses the water contamination level and alerts the administrators when it reaches a threshold. Aazam et al [16] suggests an intelligent IoT system for waste handling. It includes various sensors to capture the real time data and is updated oin the central cloud database. The bin status updation is sent to the administrator whenever the bin content reaches a threshold value. The separate values based on waste category are provided in this system which may efficiently enact the waste disposal.

Fariha Irfan Khan et al [17] proposed a system that shuts the lid of the bin when the bin gets completely filled. Ultrasonic distance calculation sensors are been used for checking bin status. When the lid is shut, a message is been send to the server side and the remaining process is been carried out by them. A database of the truck driver is been maintained that contains the information of the truck's location. Location of the truck driver can be identified through the GPS installed on the truck. Once the bin gets filled, alert message will be sent to truck drivers. If another bin which is also gets filled, and produce an alert message which is nearer to the truck driver, he should collect that waste also. Therefore, larger trucks are been used for collecting waste

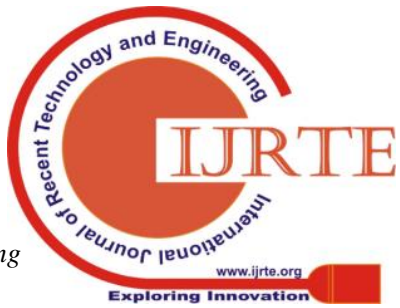


from several bins. When a truck gets fully filled, then no more alert messages will be sent to that truck driver even though the bin is nearer to that truck.

Poddar et al [18] proposed a smart bin which automatically opens its lid when a human presence is detected in front of it. The ultrasonic sensor senses the bin level and updates the real time bin fill rate to the waste collector. It also provides the optimized path for the waste collector the filled bins. This system is more efficient and economical as it uses optimized path. Wijaya et al [19] uses load calibration method to measure the bin waste and thereby estimates the waste content in the smart bin. Thus it doesn't require any external devices or components to measure the bin level. Upadhyay et al [20] proposed an integrated cloud based IoT system for waste management in smart cities. It measures humidity, temperature, fire detection using suitable sensors and update the same to the central processor. Communication protocols include TCP/IP and GSM/GPRS. The waste content in the bin is sensed using ultrasonic sensors. The collected information is updated in the cloud which is constantly monitored by the authorities. It is an economical efficient model for smart cities.

Most of the above mentioned work uses similar model for waste management. Ultrasonic sensor is used in most systems to measure the bin content level. But the problem of locating unfilled dust bins nearby for a user is not implemented in most systems.

\section{PROPOSED SYSTEM}

Our proposed system consists of three modules as shown in Figure 2: A smart dustbin, a central coordinator and an Android application. The smart bin comprises of a GSM module, ultrasonic sensor, PIR sensor and a servo motor integrated to a central coordinator system. These components acts as the server side of our proposed system. The key purpose of ultrasonic sensor is to estimate the waste level inside bin by calculating the distance from the bin top to the waste level. The sensed value is read by the central coordinator and calculates the equivalent distances in centimeters. When the garbage level in the bin reaches a threshold, an alert message will be sent to the garbage collector using Android application. The application also provides the shortest optimal path from the dust pin to the current location of the garbage collector using genetic algorithm. The smart bin produces a buzzer alarm when it is full and thereby controls the overflow. The central coordinator module consists of user module and waste collector module.

\section{A. Android Application}

The Android App has two modes: A User mode and a Garbage Collector mode. In user mode, the end user can search for the nearby dustbin which is free to put the garbage. It provides the shortest route to the bin from the user's current location. In the Garbage collector mode, it alerts the garbage collector by initiating an alarm message when the garbage level reaches a threshold. The application tracks the current location of garbage collector and shows the optimized route to all the dust bins which attained its threshold. The application also provides facility to the garbage collector to remotely check the bin status whenever required. GSM module inserted with a sim is used for the communication.

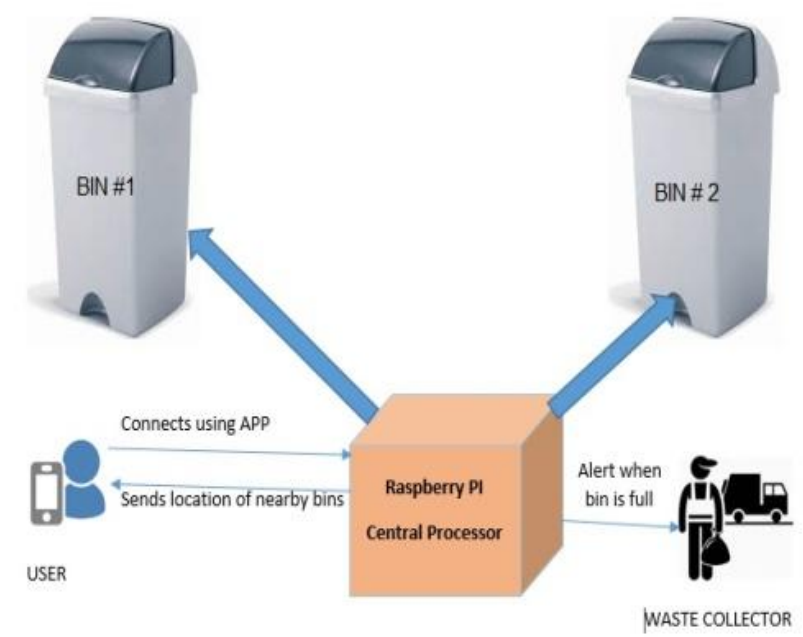

Figure 2: Proposed System Architecture

\section{B. Smart dust bin}

An ultrasonic sensor is fixed at the top of the smart dust bin. The sensor produces sound pulses of high frequency which will hit on the garbage and echo back. The time taken to reflect back is analyzed and converted to the equivalent distance in centimeters. If Total_Bin_distance denotes the height of the bin and Empty_distance represents the free space in the bin then the Garbage_level can be estimated as follows:

Garbage_level=Total_Bin_distance - Empty_distance

The percentage of garbage in the smart bin can be calculated using the following equation.

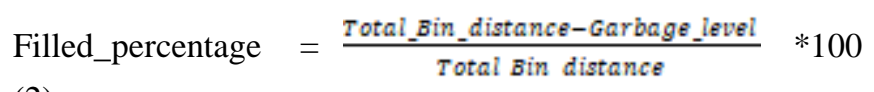
(2)



Figure 3: Smart Bin

\section{Central coordinator}

The central coordinator is a Arduino system [21] which has powerful processing capability as well as having $\mathrm{Wi}-\mathrm{Fi}$ module and interfaces. The central coordinator controls and coordinates all the activities in this project. The smart bin is automatically operated with control of Arduino. The

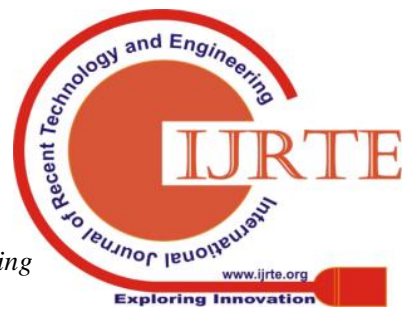


sensed bin level is fed to the central processor. When the central processor [22] exceeds a threshold value, it alerts the waste collector through email / SMS. Central coordinator acts as server side; all the data collected and stored in the database. It also consists of an optimal trip planner and bin placement planner using Travelling Salesman Algorithm is provided below.

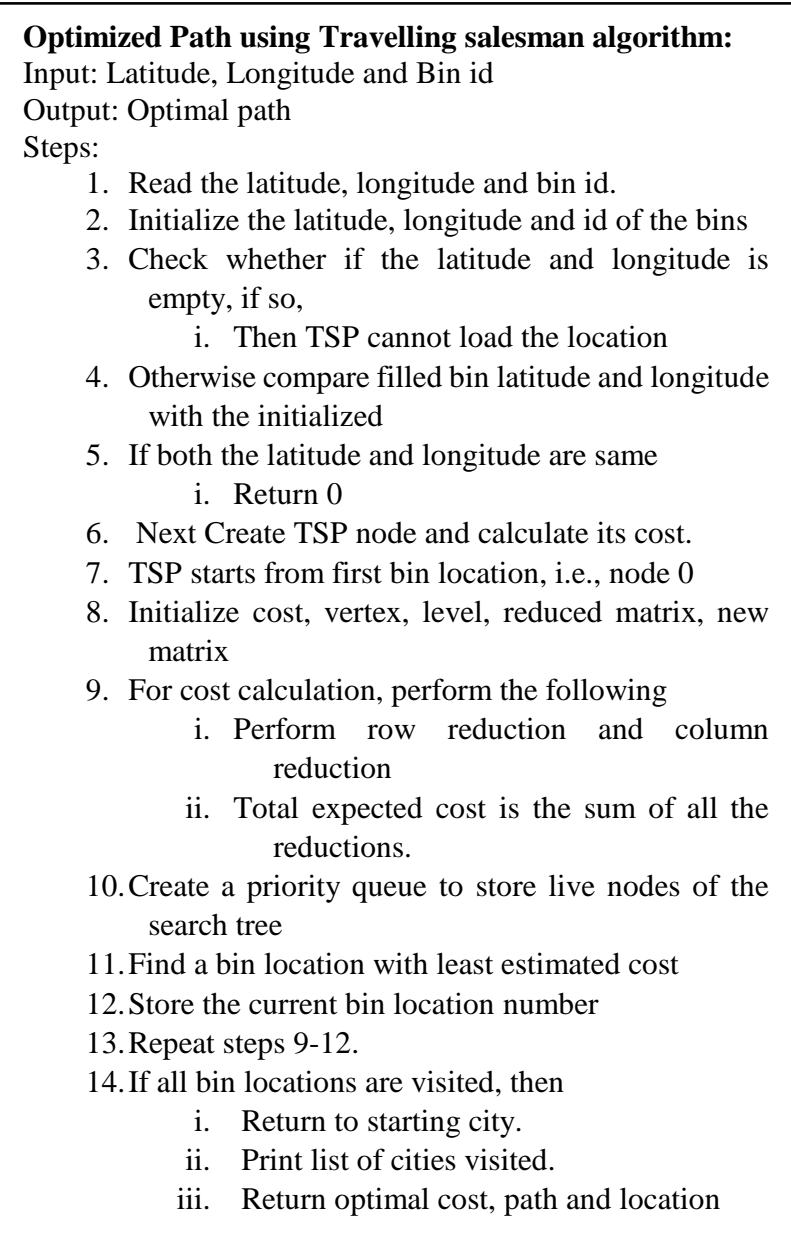

The system work flow from both client side and server side is illustrated in Figure 4.

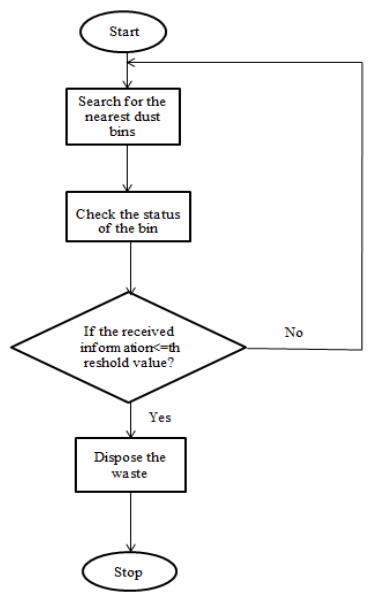

Client Side

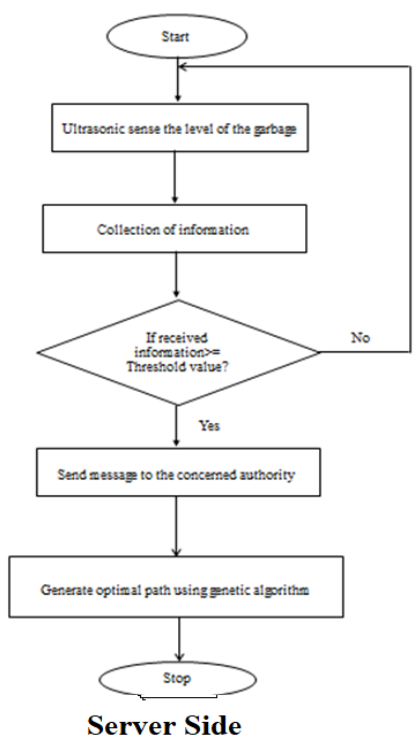

Server Side

Figure 4: Client side and server side flow chart

\section{Dear Waste Collector, Please clear the bin at location: Providence College \\ Dear Waste Collector, Please clear the bin at location: Providence College \\ Dear Waste Collector, Please clear the bin at location: Providence College \\ Dear Waste Collector, Please clear the bin at location: Providence College}

Figure 6: Android application - Bin level notification

The Android app is installed the user mobile. When the user searches for the nearby bins, the application searches for the nearby bin locations and identifies the GPS coordinates of bin locations. With the help of Google map, the application provides the route towards the bin in the user's mobile as 
shown in the left part of Figure 7. Travelling Salesman algorithm is used to generate the optimal path from the garbage collector to the dust bin. Product is validated using various testing strategies [23].

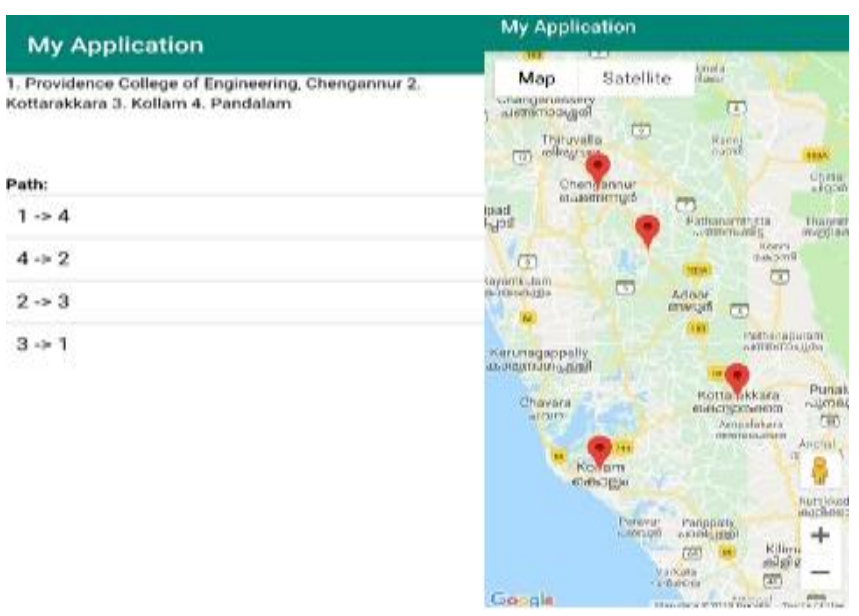

Figure 7: Optimal path to bin locations

The advantages of our proposed system are:

- More user friendly and easier to locate nearby bins.

- Average time spent by each garbage collection vehicle during a time period can be minimized.

- Average route covered by each garbage collection vehicle an be improved.

- Auto alerting about the bin condition to the waste collector.

- More economical and optimum utilization of resources.

- Dynamic routing enhances efficiency.

\section{CONCLUSIONS}

Our proposed smart waste management system consists of a smart bin and a mobile application controlled by a central coordinator. The mobile application helps the user to identify the nearby bin locations. Travelling Salesman algorithm is used to find the optimal path for the same. The sensors incorporated in the bin identify the bin status and alerts the garbage collector when the bin level reaches a threshold value. Thus our smart waste management system helps the authorities to save time as well as cost. It also reduces human effort and manages wastes in a healthier way. Our future work aims to address the automation of waste treatment plant in an efficient and economical manner.

\section{ACKNOWLEDGMENT}

We express our sincere gratitude to Providence College of Engineering, Chengannur, Kerala for the support provided. We are thankful to Mr. Sreejith P and Ms. Jisha Elizabeth Mammen for the technical support provided in executing test cases in the laboratory.

\section{REFERENCES}

[1] Sahil Mirchandani, Sagar Wadhwa, Preeti Wadhwa, Richard Joseph," IoT Enabled Dustbins", 2017 International Conference on Big Data, IoT and Data Science (BID) Vishwakarma Institute of Technology, Pune, Dec 20-22, 2017.
[2] Harshita Chugh, Dushyant singh, Shahensha shaik, Ashwani Singla,' IOT Based Smart Bin”, International Research Journal of Engineering and Technology (IRJET) Volume: 04 Issue: 09 |Sep -2017

[3] Chandradeep Tiwari, Smt. Nagarathna. K,"_Waste Management using Solar Smart Bin", International Conference on Energy, Communication, Data Analytics and Soft Computing (ICECDS-2017).

[4] S.Karthikeyan. G.Sheela Rani M.Sridevi, P.T.V.Bhuvaneswari, "IoT enabled Waste Management System using ZigBee Network", 2017 2nd IEEE International Conference On Recent Trends in Electronics Information \& Communication Technology (RTEICT), May 19-20, 2017.

[5] Prof. B.S.Malapur ,Vani R.Pattanshetti(PG), "IoT based Waste Management : An Application to Smart City”,International Conference on Energy, Communication,Data Analytics and Soft Computing,2017.

[6] Abhimanyu Singh, Pankhuri Aggarwal, Rahul Arora, "IoT based Waste Collection System using Infrared Sensors", 2016 5th International Conference on Reliability, Infocom Technologies and Optimization (ICRITO) (Trends and Future Directions), Sep. 7-9-2016,AIIT, Amity University Uttar Pradesh,Noida,India.

[7] Ujwala Ravale, Anindita Khade, Namrata Patel, Suvarna Chaure,' SMART TRASH: An Efficient Way for Monitoring Solid Waste Management", International Conference on Current Trends in Computer, Electrical, Electronics and Communication (ICCTCEEC-2017).

[8] K. Jayalakshmi, S. Pavithra, C. Aarthi," Waste to Wealth-A Novel Approach for Food Waste Management", 2017 International Conference on Electrical, Instrumentation and Communication Engineering,2017.

[9] Sudharani Ashok Ghadage, Dr. Mrs. Neeta Anilkumar Doshi," IoT Based Garbage Management (Monitor and Acknowledgment) System: A Review ", Proceedings of the International Conference on Intelligent Sustainable Systems (ICISS 2017)

[10] Sam Aleyadeh, Abd-Elhamid M. Taha." An IoT-based Architecture for Waste Management".

[11] Maneesha V Ramesh, Nibi K V , Anupama Kurup , Renjith Mohan ,Aiswarya A, Arsha A, Sarang P R , "water quality monotoring and waste management using IoT".

[12] Mustafa M.R, and Ku Azir K.N.F , "Smart Bin: Internet-of-Things Garbage Monitoring System", MATEC Web of Conferences 140, 01030 (2017).

[13] Cyril Joe Baby, Harvir Singh, Archit Srivastava, Ritwik Dhawan and P. Mahalakshmi ," Smart Bin: An Intelligent Waste Alert and Prediction System Using Machine Learning Approach”, IEEE WiSPNET 2017 conference.

[14] Neetha, Sanjana Sharma, Vaishnavi V,Vandana Bedhi, "Smart BinAn "Internet of Things" Approach to Clean and safe Public Space", International conference on I-SMAC (IoT in Social, Mobile, Analytics and Cloud) (I-SMAC 2017).

[15] Theodoros Anagnostopoulos, Arkady Zaslavsky, Alexey Medvedev, Sergei Khoruzhnicov, "Top-k Query based Dynamic Scheduling for IoT-enabled Smart City Waste Collection”, 2015 16th IEEE International Conference on Mobile Data Management.

[16] Fariha Irfan Khan and Prof. Aruna Gawade, "Dynamic Routing for Waste Management usingIoT for Cost- Efficient Service", International Conference on Current Trends in Computer, Electrical, Electronics and Communication (ICCTCEEC-2017).

[17] Hitesh Poddar, Rituraj Paul, Sourangsu Mukherjee, Budhaditya Bhattacharyya , "Design Of Smart Bin For Smarter Cities" International Conference on Innovations in Power and Advanced Computing Technologies [i-PACT2017].

[18] Mohd. Talha Amar Upadhyay Raaziyah Shamim M. Salim Beg, "A Cloud integrated wireless garbage management system for Smart Cities", IMPACT 2017.

[19] Mohammad Aazam, Marc St- Hilaire, Chung- Horng Lung, Ioannis Lambadaris, "Cloud-based Smart Waste Management for Smart Cities".

[20] Aksan Surya Wijaya , Zahir Zainuddin , Muhammad Niswar ," Design a Smart Waste Bin for Smart Waste Management", 2017 th International Conference on Instrumentation, Control, and Automation (ICA), Yogyakarta,Indonesia.

[21] P. M. Jacob and P. Mani, "A Reference Model for Testing Internet of Things based Applications", Journal of 
Engineering, Science and Technology (JESTEC), Vol. 13, No. 8 (2018), pp. 2504-2519.

[22] P. M. Jacob and P. Mani, "Software architecture pattern selection model for Internet of Things based systems," in IET Software, vol. 12, no. 5, pp. 390-396, 10 2018. doi: 10.1049/iet-sen.2017.0206.

[23] Pramod Mathew Jacob and M. Prasanna, "A Comparative analysis on black box testing strategies," International Conference on Information Science - ICIS -'16, Kochi, India, 2016

\section{AUTHORS PROFILE}

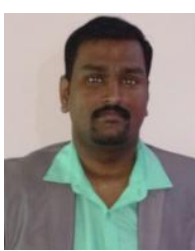

Pramod Mathew Jacob has completed his B. Tech in Computer Science \& Engineering from Kerala University. He possesses M. Tech in Software Engineering from SRM Institute of Science and Technology Chennai. He is working as Assistant Professor in Providence College of Engineering, Kerala. Presently he is pursuing PhD in Vellore Institute of Technology Vellore. He has a teaching experience of 5 years and Research experience of two years. He has published 8 papers in various international journals and conferences. His areas of interest include Software Engineering, Software Testing, and Internet of Things.

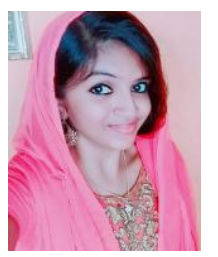

Rasha Kabeer has completed her B. Tech in Computer Science \& Engineering from APJ Abdul Kalam Technological University in Kerala. She has done her graduation from Providence College of Engineering, Chengannur. Her areas of interest include Internet of Things and Mobile applications development.

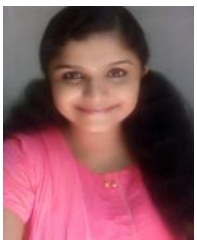

Negha Nair has completed her B. Tech in Computer Science \& Engineering from APJ Abdul Kalam Technological University in Kerala. She has done her graduation from Providence College of Engineering, Chengannur. Her areas of interest include Internet of Things, Computer Networks, Web designing etc.

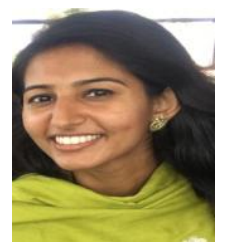

Sony V Saji has completed her B. Tech in Computer Science \& Engineering from APJ Abdul Kalam Technological University in Kerala. She has done her graduation from Providence College of Engineering, Chengannur. Her areas of interest include Internet of Things and Smart computing.



Meenakshi has completed her B. Tech in Computer Science \& Engineering from APJ Abdul Kalam Technological University in Kerala. She has done her graduation from Providence College of Engineering, Chengannur. Her areas of interest include Internet of Things and Computer Networks.

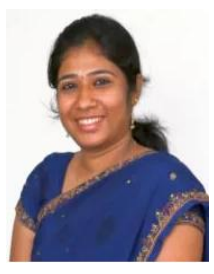

Renju Rachel Varghese has completed her B. Tech in Computer Science \& Engineering from Cochin University. She possesses M. Tech in Image Processing from CUSAT, Kerala. She is working as Assistant Professor in Providence College of Engineering, Kerala. She has a teaching experience of 3 years and her areas of interest include Image Processing, Data mining, Machine Learning and Internet of Things. 